\title{
Effect of dietary supplementation with Spirulina on the expressions of AANAT, ADRB3, BTG2 and FASN genes in the subcutaneous adipose and Longissimus dorsi muscle tissues of purebred and crossbred Australian sheep
}

Arash Kashani ${ }^{1}$, Benjamin William Behrens Holman², Peter David Nichols ${ }^{3}$ and Aduli Enoch Othniel Malau-Aduli ${ }^{1,4^{*}}$

\begin{abstract}
Background: The demand for healthy, lean and consistent meat products containing low saturated fatty acid content and high quality polyunsaturated fatty acids (PUFA), especially long-chain ( $\geq C_{20}$ ) omega-3 PUFA, has increased in recent times. Fat deposition is altered by both the genetic background and dietary supplements, and this study aimed to assess the effect of dietary Spirulina supplementation levels on the mRNA expression patterns of genes controlling lipid metabolism in the subcutaneous adipose tissue (SAT) and Longissimus dorsi (Id) muscle of Australian crossbred sheep.

Methods: Twenty-four weaned lambs belonging to four breeds under the same management conditions were maintained on ryegrass pasture and fed three levels of Spirulina supplement (control, low and high). In terms of nutrient composition, Spirulina is a nutrient-rich supplement that contains all essential amino acids, vitamins and minerals. It also is a rich source of carotenoids and fatty acids, especially gamma-linolenic acid (GLA) that infer health benefits. After slaughter, subcutaneous adipose tissue (SAT) and Id samples were subjected to mRNA extraction and reverse transcription using quantitative polymerase chain reaction (RT-qPCR) to assess the mRNA expression levels of the Aralkylamine N-acetyltransferase (AANAT), Adrenergic beta-3 receptor (ADRB3), B-cell translocation gene 2 (BTG2) and Fatty acid synthase (FASN) genes, which are associated with lipid metabolism.

Results: Both low and high Spirulina supplementation levels strongly up-regulated the transcription of all the selected genes in both SAT and Id tissues (mostly in the subcutaneous adipose), but sheep breed and sex did not influence the gene expression patterns in these tissues.

Conclusions: The evidence indicates that high Spirulina supplementation level resulted in a decrease in intramuscular fat content in Australian purebred and crossbred sheep due to the enhanced production of melatonin in sheep muscle tissues and strong up-regulation of mRNA expression of BTG2 in SAT which negatively affected fat deposition. In contrast, low Spirulina supplementation level strongly up-regulated the ADRB3 and FASN genes responsible for fat production. These findings are consistent with the observed phenotypic data suggesting that low Spirulina supplementation level can increase lamb production, with higher long-chain PUFA content.
\end{abstract}

\footnotetext{
* Correspondence: Aduli.MalauAduli@utas.edu.au

'Animal Science and Genetics, Tasmanian Institute of Agriculture, School of Land and Food, Faculty of Science, Engineering and Technology, University of Tasmania, Private Bag 54 Sandy Bay, Hobart, Tasmania 7001, Australia ${ }^{4}$ Veterinary and Biomedical Sciences, College of Public Health, Medical and Veterinary Sciences, Division of Tropical Health and Medicine, James Cook University, Townsville, Queensland 4811, Australia

Full list of author information is available at the end of the article
} 


\section{Background}

Inclusion of marine supplements in the diet of sheep represents an effective nutritional strategy for altering meat production and enhancing polyunsaturated fatty acids (PUFA) [1,2]. Spirulina (Arthrospira platensis) is an edible blue-green microalga, highly nutritious supplement containing 60 to $70 \%$ protein and is a potential feed resource for many animal species. Research findings have linked Spirulina to an improvement in animal growth and nutritional product quality [2-4].

Meat with superior eating qualities and healthier nutritional composition commands a higher price that consumers are generally prepared to pay. Traditionally, the fat content of meat has been considered as an important source of essential fatty acids and as a calorie-dense nutrient [5]. Within the last decade, fats, and particularly fatty acids, have been increasingly recognised as major biological regulators of the quality of fresh meat and sensory value of meat products [6,7]. In addition, fatty acids can influence sterol metabolism, signal transduction, enzyme activities, cell proliferation, differentiation and receptor expression [5,6]. The cellular effect of fatty acids is related to the regulation of gene expression and subsequent downstream events, and omega- 3 fatty acids are especially potent in affecting many of the metabolic pathways [6].

Fat deposition and composition can be altered by the animal's genetic background and dietary supplements fed, thus indicating that both factors have significant influences on adipogenic and lipogenic metabolic pathways [8]. However, the molecular mechanisms underlying fat deposition and fatty acid composition in sheep are not yet fully understood. To our knowledge, there is only limited available information addressing the molecular adaptation of ovine tissues to supplementation with dietary marine ingredients that induce superior quality of meat production and PUFA enhancement.

This study aimed to investigate the changes in mRNA expression patterns of these key genes: Aralkylamine $N$-acetyltransferase (AANAT), Adrenergic beta-3 receptor (ADRB3), B-cell translocation gene 2 (BTG2), and Fatty acid synthase (FASN) controlling lipid metabolism in subcutaneous adipose tissue (SAT) and Longissimus dorsi $(l d)$ muscle of Australian purebred and crossbred lambs subjected to zero, low and high levels of dietary supplementation with Spirulina.

\section{Methods}

\section{Experimental animals and data collection}

The use of animals and procedures performed in this study were all approved by the University of Tasmania Animal Ethics Committee, and were conducted in accordance with the 1993 Tasmanian Animal Welfare Act and the 2004 Australian Code of Practice for the Care and Use of Animals for Scientific Purposes. The design of the experiment has been described previously [2]. Briefly, 24 crossbred lambs comprising 12 wethers and 12 ewes of purebred Merino, Black Suffolk x Merino, Dorset x Merino and White Suffolk x Merino crossbred lambs were randomly assigned into three supplementary treatment groups: the control group grazing without Spirulina (0\%), low $(100 \mathrm{~mL} / \mathrm{head} /$ day in the ratio of $1 \mathrm{~g}$ of Spirulina powder:10mL of water or $10 \% \mathrm{wt} / \mathrm{vol}$ ), and high $(200 \mathrm{~mL} / \mathrm{head} /$ day in the ratio of $2 \mathrm{~g}$ of Spirulina powder: $10 \mathrm{~mL}$ of water or $20 \% \mathrm{wt} / \mathrm{vol}$ ) Spirulina supplementation levels. The Spirulina powder was purchased from a commercial producer in Darwin, Northern Territory, Australia (TAAU, NT, Aus). Lambs were daily supplemented according to their assigned Spirulina treatment group before being released into paddocks for grazing. All lambs had ad libitum access to clean drinking water and ryegrass pastures. Following slaughter after nine weeks of the feeding trial, approximately $10 \mathrm{~g}$ of subcutaneous adipose and Longissimus dorsi muscle tissues were collected from the carcass and frozen for subsequent RNA extraction and further analysis.

\section{RNA extraction and CDNA synthesis}

Total RNA was isolated from the thawed frozen tissues using TRIzol ${ }^{\circ}$ Plus RNA Purification Kit (Life Technologies Pty Ltd. Victoria, Australia). Homogenisation of the sample in TRIzol $^{\circ}$ reagent was performed using a tissue lyser (Qiagen Ltd., Crawley, UK). RNA was extracted using chloroform and precipitated using isopropanol. The quantity of total RNA extracted was assessed using the NanoDrop 8000 spectrophotometer (NanoDrop, Wilmington, DE). RNA quality was verified by ensuring that all RNA samples had an absorbance ratio (A260/280) between 1.8 and 2. RNA samples were treated with PureLink ${ }^{\mathrm{mm}}$ DNase (Life Technologies Pty Ltd. Victoria, Australia) and purified using the RNeasy1 Mini Kit (Qiagen Ltd.). DNase-treated and purified total RNA was then reverse transcribed to cDNA with Mixed Oligo dT/Random Hexamer Primers using the Tetro cDNA Synthesis Kit (Bioline Pty Ltd. NSW, Australia) according to the manufacturer's instructions and stored at $-80^{\circ} \mathrm{C}$ for subsequent analyses.

\section{Primer design and reference gene selection}

All candidate and reference gene primers used for gene expression (Table 1) were designed using the Primer3 web based software program (http://frodo.wi.mit.edu/ primer3/), and obtained from a commercial supplier (GeneWorks Pty Ltd., SA, Australia). Primer specificity was checked using the Basic Local Alignment Search Tool (BLAST) at the National Center for Biotechnology Information (http://www.ncbi.nlm.nih.gov/BLAST/). All primers were validated using pooled cDNA samples. A 
standard curve was generated using serial dilutions of pooled cDNA. PCR products generated by amplification were sequenced to verify their primer specific identity (Beckman Coulter CEQ $^{\mathrm{m}} 8000$ Series Genetic Analysis System, University Tasmania). To determine the relative gene expression levels, suitable and highly stable reference genes were required. A total of five reference genes were tested out of which two [Ubiquitin $C(U B C)$ and Peptidylprolyl cis-trans isomeraseA (PPIA)] were finally chosen to normalise the gene expression data for AANAT, BTG2, FASN and ADRB3 transcription levels in both tissues. The principle behind the selection of the two reference genes was their constant expression ratios across all samples. The gene expression stability ( $\mathrm{M}$-value) of the reference genes was calculated and validated using the geNorm software (version 3.5).

\section{Quantitative real time PCR (qPCR)}

Following reverse transcription, cDNA quantity was determined and standardised to the required concentration for qPCR. Triplicate $20 \mu \mathrm{L}$ reactions were carried out in a 72-well Rotor-Gene (QIAGEN GmbH, Hilden, Germany), containing $4 \mu \mathrm{L}$ cDNA (50 ng), $10 \mu \mathrm{L} 2 \times$ SensiFAST SYBR No-ROX Mix (Bioline Pty Ltd., NSW, Australia), $4.4 \mu \mathrm{L}$ DEPC $\mathrm{H}_{2} \mathrm{O}$, and $0.8 \mu \mathrm{L}$ forward and reverse primers (100 fmol). Assays were performed using the Rotor-Gene 3000 (QIAGEN Pty Ltd., VIC, Australia) with the following cycling parameters: $95^{\circ} \mathrm{C}$ for $2 \mathrm{~min}$ polymerase activation; 40 cycles of $95^{\circ} \mathrm{C}$ for $5 \mathrm{~s}$ denaturation, $60^{\circ} \mathrm{C}$ for $10 \mathrm{~s}$ annealing and $72^{\circ} \mathrm{C}$ for $5 \mathrm{~s}$ extension. Gene expression levels were recorded as $\mathrm{Ct}$ values (i.e., the number of PCR cycles at which the fluorescence signal was detected above the threshold value) and all samples were run in triplicates. Amplification efficiencies were determined for all candidate and reference genes using the formula $E=10^{\wedge}$ (-1/slope), with the slope of the linear curve of cycle threshold $(\mathrm{Ct})$ values plotted against the log dilution [9] (Higuchi et al., 1993). Primer concentrations were optimised for each gene and disassociation curves were examined for the presence of a single PCR product. The efficiency of the reaction was calculated using a 5-fold serial dilution of cDNA and generation of a standard curve. All PCR efficiency coefficients were between 1.7 and 1.8 and therefore deemed acceptable. The software package Rotor-Gene 3000 (version 6.0.16) (QIAGEN Pty Ltd., VIC, Australia) was used for efficiency correction of the raw $\mathrm{Ct}$ values. This process involved an inter-plate calibration based on a calibrator sample included on all plates, averaging of replicates, normalisation to the reference gene and the calculation of quantities relative to the highest $\mathrm{Ct}$ and $\log 2$ transformation of the expression values for all genes.

\section{Statistical analysis}

A generalised linear model (GLM) (SAS Inst., NC) was used in computing the fixed effects of Spirulina supplementation level, sire breed and sex, and their interactions on mRNA expression level of AANAT, ADRB3, BTG2 and $F A S N$ genes in subcutaneous adipose and muscle tissues. Separation of significant least squares means was tested at a minimum of $5 \%$ threshold using Tukey's pairwise comparisons.

\section{Results}

\section{Spirulina supplementation and phenotypic data}

The candidate and reference gene primers used for the gene expression analysis are shown in Table 1. Spirulina supplementation enabled sheep to grow longer bodies (BL) than the control group $(P<0.015)$ as portrayed in Table 2. Furthermore, lambs in the high Spirulina supplementation treatment group had greater body condition score (BCS) than the low and control treatment groups $(P<0.001)$. It was observed that sheep receiving low Spirulina supplementation had the heaviest body weight $(\mathrm{BWT})$ of $41.9 \mathrm{~kg}(P<0.018)$. However, no differences were observed between the high and control treatment groups. The phenotypic results are shown in Table 2.

Table 1 Primer pairs designed for real-time PCR (qPCR)

\begin{tabular}{|c|c|c|c|c|}
\hline${ }^{\mathrm{a} G e n e}$ symbol & qPCR primers & & ${ }^{\mathrm{b}} \mathrm{T}_{\mathrm{a}}$ & Amplicon size (bp) \\
\hline & Forward Primer & Reverse Primer & & \\
\hline AANAT & ACTGACCTTCACGGAGATGC & TTCACTCATTCTCCCCGTTC & 60 & 211 \\
\hline ADRB3 & TCAGTAGGAAGCGGGTCGGG & GGCTGGGGAAGGGCAGAGTT & 60 & 291 \\
\hline BTG2 & CTGGAGGAGAACTGGCTGTC & AAAACAATGCCCAAGGTCTG & 60 & 194 \\
\hline FASN & GTGTGGTACAGCCCCTCAAG & ACGCACCTGAATGACCACTT & 60 & 110 \\
\hline \multicolumn{5}{|c|}{ Reference genes } \\
\hline UBC & CGTCTTAGGGGTGGCTGTTA & AAATTGGGGTAAATGGCTAGA & 60 & 90 \\
\hline PPIA & TCATTTGCACTGCCAAGACTG & TCATGCCCTCTTTCACTTTGC & 60 & 72 \\
\hline
\end{tabular}

${ }^{\mathrm{a}}$ Aralkylamine $\mathrm{N}$-acetyltransferase $=$ AANAT, $\beta 3$-adrenergic receptor $=$ ADRB3, B-cell translocation gene $2=\mathrm{BTG} 2$, Fatty acid synthase $=\mathrm{FASN}, \mathrm{Ubiquitin} C=\mathrm{UBC}$, Peptidyl-prolyl cis-trans isomerase $\mathrm{A}=\mathrm{PPIA},{ }^{b} \mathrm{Ta}=$ Empirical annealing Temperature. 


\section{Gene expression pattern}

To determine the expression patterns of AANAT, $A D R B 3, B T G 2$ and FASN genes, a panel of subcutaneous and adipose tissues was collected from 20 genetically divergent Australian purebred and crossbred sheep supplemented with either zero, low or high levels of Spirulina. The genes were investigated directly for mRNA expression by qRT-PCR molecular biology techniques. The qRT-PCR results were calibrated and normalized using two housekeeping genes (UBC and PPIA) and the qBase relative quantification excel application [10] for automated analysis.

\section{Gene expression in the subcutaneous adipose tissue}

The relative mRNA expression levels of AANAT, $A D R B 3, B T G 2$ and FASN genes analysed in the subcutaneous adipose tissue (SAT) are presented in Figure 1. $A D R B 3, B T G 2$ and $F A S N$ showed higher expression levels in tissues from sheep that received low or high levels of Spirulina supplementation, relative to the control. In contrast, Spirulina supplementation did not alter the mRNA expression of AANAT gene in SAT.

Ten percent dietary Spirulina supplementation upregulated mRNA expression of the ADRB3 gene $(P<$ 0.001 ), corresponding to a 5.27 folds change compared to the control treatment (Figure 1), whereas high Spirulina supplementation resulted in a down-regulation of $A D R B 3$ mRNA level, which is 5.08 -folds lesser than the low Spirulina supplementation treatment and similar to the control group (1.19-folds higher). The mRNA expression level of BTG2 gene was significantly higher $(P<0.001)$ in tissues from sheep supplemented with high levels of Spirulina compared to either the $10 \%$ or control conditions (Figure 1). Low Spirulina dietary supplementation resulted in an up-regulation of BTG2 mRNA levels (0.83-folds) compared to the control, but this was not statistically significantly. However, high Spirulina supplementation resulted in a significantly higher expression level (7.95-folds increase) in SAT compared to control. A similar expression profile was observed for the FASN gene as dietary Spirulina up-regulated the mRNA expression levels in both the low and high supplementation groups; FASN expression increased 8.4-folds $(P<0.05)$ under the low supplementation treatment group and 10.06-folds under the high supplementation level, relative to control treatment (Figure 1).

\section{Gene expression in the Longissimus dorsi muscle}

The relative mRNA expression levels of the four genes in the Longissimus dorsi $(l d)$ muscle tissue are presented in Figure 2. It was evident that under both the low and high dietary Spirulina supplementation groups, the mRNA expression levels of AANAT gene increased. However, this increase was only statistically significant in the high Spirulina supplementation treatment group, in which the AANAT mRNA expression level was 19.83folds up-regulated compared to the control. Spirulina supplementation had no significant effect $(P>0.05)$ on the mRNA levels of $A D R B 3, B T G 2$ and FASN genes (Figure 2). However, in the high Spirulina supplementation group, there was an up-regulation in the BTG2 (0.76-folds) and FASN (1.28-folds) mRNA levels compared to the control.

\section{Discussion}

Fat and fatty acid deposition in animal tissues has been attributed to a complex regulation network of lipogenic genes, genetic differences, and diet. However, the molecular mechanisms underlying these systems remain to be fully understood and characterized. Therefore, understanding the factors affecting the depot-specific fat accretion and metabolism in sheep is of paramount importance. The present study addressed these aspects based on an experimental trial with four genetically diverse sheep breeds and three levels of protein-rich dietary supplementation with Spirulina. The results reported here indicate that genetic background (sire breed) and to a lesser extent sex, determined the mRNA

Table 2 Least square means (LSM) of average daily gain, body conformation, condition score and liveweight in Spirulina supplemented crossbred lambs

\begin{tabular}{|c|c|c|c|c|c|c|c|c|c|c|c|c|}
\hline & Spirulina & & & Breed & & & & Sex & & $P$ values & & \\
\hline & Control & Low & High & $B S$ & WS & D & $M$ & W & $E$ & Spirulina & Breed & Sex \\
\hline $\mathrm{CG}(\mathrm{cm})$ & 95.0 & 95.6 & 96.1 & $99.0^{a}$ & $94.4^{b}$ & $93.8^{b}$ & $95.0^{\mathrm{b}}$ & $96.2^{a}$ & $94.9^{b}$ & $0.376^{\mathrm{ns}}$ & $0.001^{* * *}$ & $0.034^{*}$ \\
\hline WH $(\mathrm{cm})$ & 62.9 & 62.7 & 63.1 & $63.6^{a}$ & $62.8^{a}$ & $63.5^{a}$ & $61.6^{\mathrm{b}}$ & $63.4^{\mathrm{a}}$ & $62.4^{b}$ & $0.669^{\text {ns }}$ & $0.001^{* * *}$ & $0.009^{* *}$ \\
\hline $\mathrm{BL}(\mathrm{cm})$ & $65.7^{b}$ & $66.6^{a}$ & $66.8^{a}$ & $68.8^{a}$ & $67.0^{\mathrm{b}}$ & $66.9^{b}$ & $62.6^{c}$ & 66.5 & 66.2 & $0.015^{*}$ & $0.001^{* * *}$ & $0.269^{\text {ns }}$ \\
\hline $\mathrm{BCS}(0-5)$ & $3.2^{b}$ & $3.3^{b}$ & $3.4^{\mathrm{a}}$ & $3.7^{\mathrm{a}}$ & $3.3^{b}$ & $3.2^{b}$ & $3.1^{c}$ & 3.3 & 3.3 & $0.001^{* * *}$ & $0.001^{* * *}$ & $0.346^{\mathrm{ns}}$ \\
\hline BWT (kg) & $40.6^{b}$ & $41.9^{a}$ & $40.8^{b}$ & $46.3^{a}$ & $42.9^{b}$ & $41.8^{b}$ & $33.5^{c}$ & $42.1^{a}$ & $40.1^{b}$ & $0.018^{* *}$ & $0.001^{* * *}$ & $0.001^{* * *}$ \\
\hline ADG $(\mathrm{kg} / \mathrm{d})$ & 0.1 & 0.2 & 0.1 & 0.1 & 0.2 & 0.2 & 0.1 & 0.1 & 0.1 & $0.759^{\text {ns }}$ & $0.502^{\mathrm{ns}}$ & 0.605 \\
\hline
\end{tabular}

$B S=$ Black Suffolk, $W S=$ White Suffolk, $D=$ Dorset, $M=$ Merino, $W=$ Wethers, $E=$ Ewes.

Column means within a fixed effect bearing different superscripts significantly differ $(P<0.05)$. Chest girth $(C G)$, withers height $(W H)$, body length $(B L)$, body condition score $(B C S)$, body weight $(B W T)$, and average daily weight gain $(A D G)$. Level of significance: ns not significant $(P>0.05)$, * significant $(P<0.05)$, ** highly significant $(P<0.01)$, and ${ }^{* * *}$ very highly significant $(P<0.001)$. 


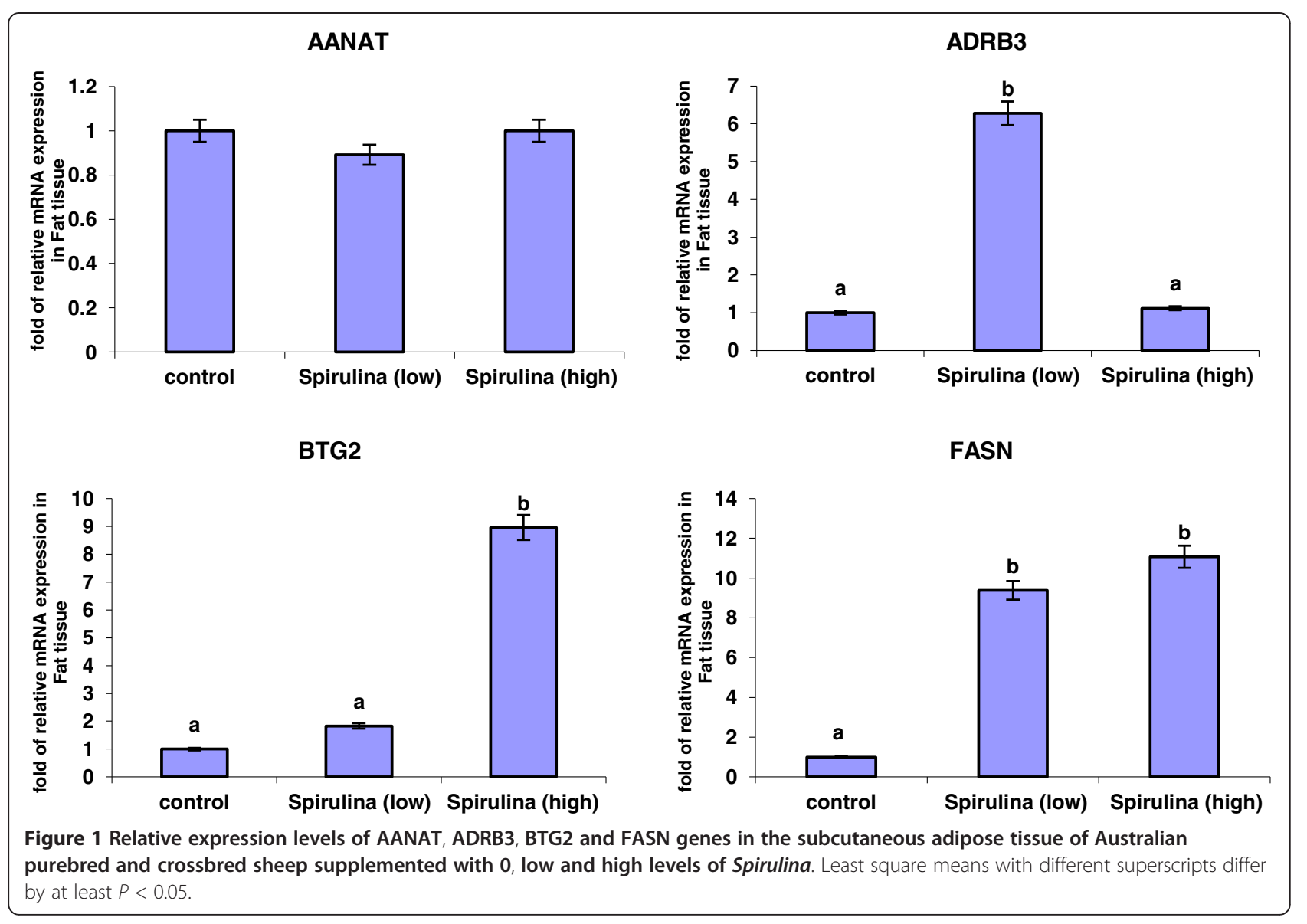

expression levels of AANAT, ADRB3, BTG2 and FASN genes. In order to elucidate the molecular mechanisms involved in this physiological process, these tissues and diet-specific variations are explained based on transcription levels of $A A N A T, A D R B 3, B T G 2$ and FASN genes.

\section{AANAT}

The AANAT gene encodes an acetyltransferase superfamily protein [11]. This acetyltransferase is the penultimate enzyme in melatonin synthesis and controls the night/day rhythm in melatonin production in the vertebrate pineal gland. The AANAT protein catalyses the rate-limiting step in the synthesis of melatonin from serotonin [7]. Melatonin is essential for the function of the circadian clock that influences activity and sleep [11,12]. AANAT transcripts have been found to be differentially expressed in high vs. low omega-3 index (O3I) muscles, suggesting a role for melatonin in reducing oxidative damage, including that to PUFA $[7,13]$.

The ability of melatonin to protect against lipid peroxidation has been repeatedly documented in many studies using animal and plant tissues [13]. Spanish scientists reported that melatonin consumption assists in the control of weight gain since it stimulates the appearance of brown fat (beige), a type of fat cell that burns calories instead of storing them [14]. Their research demonstrated that melatonin treatment not only induced browning of inguinal white adipose tissue in Zucker diabetic fatty rats, but also increased thermogenic activity [14]. Taken together, these findings highlight the anti-obesity effect of melatonin and explain its metabolic benefits of protecting against oxidative degradation of PUFA in the muscle tissue thereby producing higher O3I levels [13].

Herein, we report that $A A N A T$ transcription levels in the Longissimus dorsi muscle tissue are related to dietary Spirulina supplementation levels; high Spirulina supplementation resulted in a 7 -fold and a 20 -fold up-regulation of $A A N A T$ mRNA levels relative to the control and low Spirulina treatment groups, respectively. These findings suggest that sheep receiving the high Spirulina supplement may have lost body weight due to the enhanced production of melatonin in their muscle tissue, as observed in Table 2, and in accordance with phenotypic data from obese rats [14]. Therefore, a high level of Spirulina supplementation may be involved with, and results in, weight loss, which might occur through browning of the white adipose tissue, thus increasing the omega-3 levels in the fatty acid profile of the skeletal 


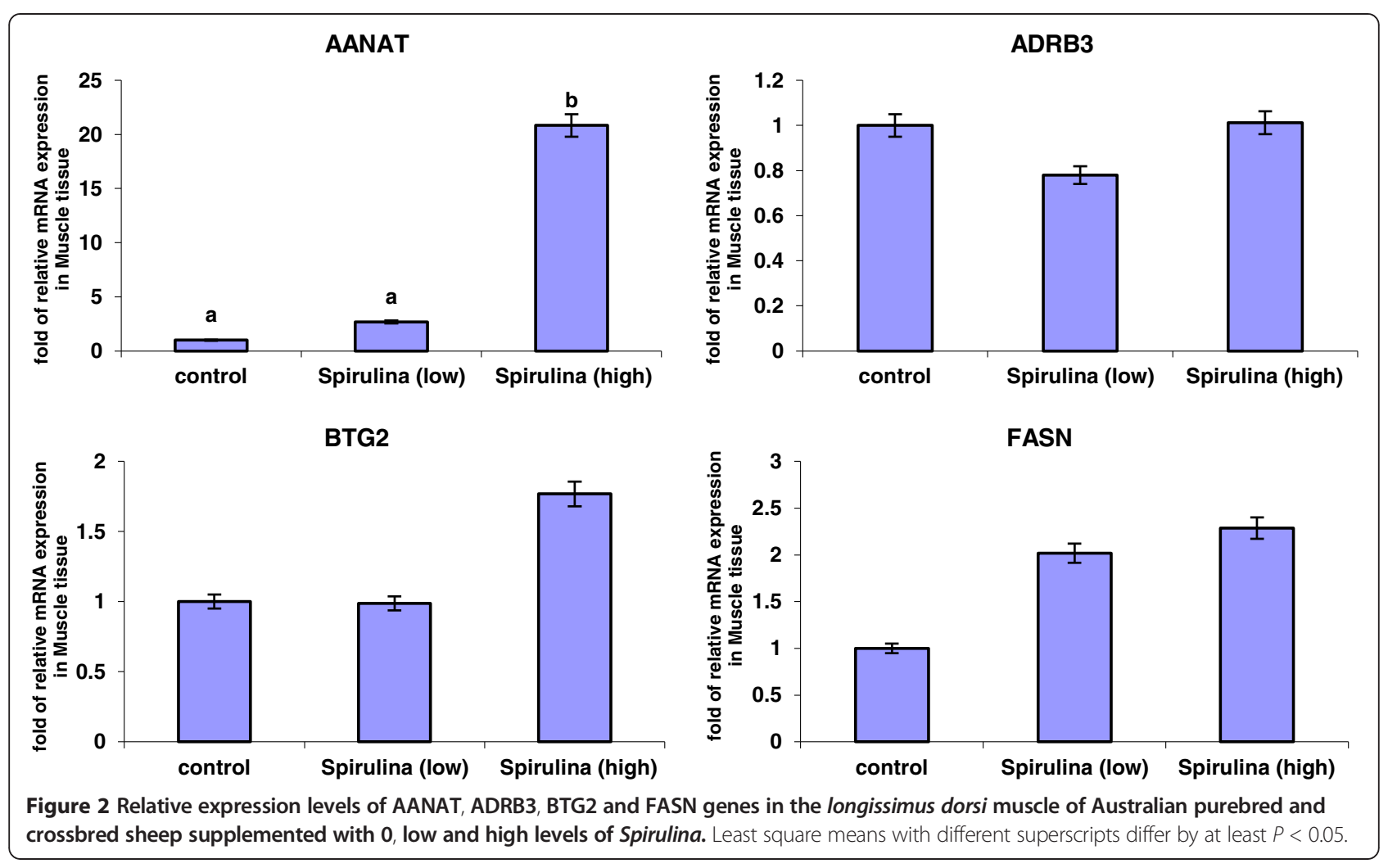

muscle tissue of sheep. However, further fatty acid analyses are required to confirm this hypothesis.

\section{ADRB3}

$A D R B 3$ encodes a protein belonging to the adrenergic receptor group of G-protein coupled receptors [15]. ADRB3 is located mainly in adipose tissue and plays a key role in regulating mammalian energy storage and expenditure under the mediating effects of the sympathetic nervous system [16,17]. ADRB3 is the principal mediator of the lipolytic and thermogenic effect of high catecholamine (in particular norepinephrine), concentration in brown and white adipose tissues in rodents $[16,18]$. The primary role of the receptor is suggested to be the regulation of resting metabolic rate and lipolysis [19].

In a large number of studies, $A D R B 3$ gene expression has been shown to be correlated with obesity in both humans and other mammals $[20,21]$. Findings from various studies now provide a consistently clear picture of the important role of $A D R B 3$ in the regulation of lipid metabolism and make this protein an obvious target for drug discovery strategies designed to treat obesity [20].

Herein, we demonstrate that $A D R B 3$ transcription levels are significantly up-regulated in SAT under low dietary Spirulina supplementation, which is consistent with our observed phenotypic results in Table 2 that confirm weight gain in this group. The ADRB3 mRNA expression levels under high Spirulina supplementation remained unchanged compared to the control group. One possible explanation for this observation is the negative correlation between protein accretion and fat deposition rates that had been exacerbated by high protein levels in feeds [2]. In addition, excess protein may become deaminated and lost in the urine or become broken down in the liver, which could result in fatty liver and ketosis [2]. Thus, we are able to speculate that the low level of Spirulina supplementation can be beneficial, giving higher production by fattening sheep in terms of early attainment of market weight. However, supplementing sheep with a higher dosage of Spirulina may result in lower efficiency in liver function and probably a decrease in total production. Further research into ADRB3 transcription levels in sheep liver would allow greater insight into the underlying biological mechanism.

\section{BTG2}

The mammalian BTG2 gene belongs to the antiproliferative (APRO) family of genes that regulate cell cycle progression in a variety of cell types [22,23]. BTG2 is a prototypical member of the $B T G / T O B$ family with antiproliferative properties. The protein encoded by this gene controls cell cycle progression and proneural gene expression by acting as a transcription co-regulator that enhances or inhibits the activity of transcription factors [22,23].

In our study, it was apparent that Spirulina supplementation increased BTG2 transcription levels in SAT. However, 
only tissues from sheep receiving a high level of Spirulina supplementation significantly over-expressed the BTG2 gene. A number of studies have demonstrated that the BTG2 gene has a potential role in muscle fibre size, intramuscular fat deposition and weight loss [22,23]. Herein, we suggest that the weight loss experienced by the high Spirulina supplementation group may be attributable to a decline of preadipocyte proliferation, an increase in energy expenditure and a decline in energy uptake in adipocytes, which may be caused by an increase in BTG2 expression.

\section{FASN}

FASN encodes a multifunctional enzyme that catalyses fatty acid synthesis [24]. FASN is considered as a fundamental enzyme in de novo lipogenesis in mammals and its main function is to catalyse the synthesis of palmitate from acetyl-CoA and malonyl-CoA, in the presence of $\mathrm{NADPH}$, into long-chain saturated fatty acids (LC-SFA) $[24,25]$. It has been shown that the FASN gene contributes to the regulation of body weight in humans, which results in the development of obesity $[24,26]$.

We demonstrate herein, that both the low and high levels of Spirulina supplementation increased the transcription levels of FASN in both SAT and muscle tissues, which may have assisted these sheep in gaining weight.

Our results demonstrate that supplementing sheep with low levels of Spirulina can increase lamb production by increasing the transcription level of $A D R B 3$ and FASN genes in SAT, and might also beneficially alter the fatty acid profile by reducing the oxidation of PUFA in skeletal muscle.

\section{Conclusions}

The results presented herein suggest that the mRNA levels of AANAT, ADRB3, BTG2 and FASN genes in the SAT and Longissimus dorsi muscle tissues are mainly influenced by dietary Spirulina level, whereas the individual effects of breed and sex, and their combined effects with diet, are not associated with the mRNA expression levels of the above genes. Taken together, our results show that lipid metabolism in SAT is more sensitive to dietary supplementation than in the muscle. These findings provide evidence to support a low to intermediate level of dietary protein supplementation for achieving optimal increase in the omega- 3 and 6 contents of red meat in Australian purebred and crossbred sheep.

\section{Competing interests}

The authors declare that they have no competing interests.

\section{Authors' contributions}

This work was carried out in collaboration between all authors. Author AK participated in the feeding trial, ran the laboratory analyses and wrote the first draft of the manuscript as part of his PhD project. Both AK and BWBH carried out the feeding trial, collected blood samples and performed feed chemical analysis. Author AEOMA and PDN conceived the research idea, wrote the funding grant, experimental design, read and made needed changes to the draft and final manuscript as a research article. All authors read and approved the final manuscript.

\section{Acknowledgements}

This research was funded by grants and postgraduate scholarships from the University of Tasmania (UTAS) and the Australian Wool Education Trust (AWET). We thank Chris Gunn, John Otto, Will Bignell and Barrie Wells for their inputs during the sheep breeding and feeding trials.

\section{Author details}

${ }^{1}$ Animal Science and Genetics, Tasmanian Institute of Agriculture, School of Land and Food, Faculty of Science, Engineering and Technology, University of Tasmania, Private Bag 54 Sandy Bay, Hobart, Tasmania 7001, Australia. ${ }^{2}$ New South Wales Department of Primary Industries, Centre for Red Meat and Sheep Development, Cowra 2794New South Wales, Australia. ${ }^{3}$ CSIRO Food and Nutrition, Oceans and Atmosphere Flagships, HobartTAS 7001 Australia. ${ }^{4}$ Veterinary and Biomedical Sciences, College of Public Health, Medical and Veterinary Sciences, Division of Tropical Health and Medicine, James Cook University, Townsville, Queensland 4811, Australia.

Received: 27 November 2014 Accepted: 12 February 2015

Published online: 04 March 2015

\section{References}

1. Berndt J, Kovacs P, Ruschke K, Klöting N, Fasshauer M, Schön MR, et al. Fatty acid synthase gene expression in human adipose tissue: association with obesity and type 2 diabetes. Diabetologia. 2007;50:1472-80.

2. Bichi E, Frutos P, Toral PG, Keisler D, Hervás G, Loor JJ. Dietary marine algae and its influence on tissue gene network expression during milk fat depression in dairy ewes. Anim Feed Sci Technol. 2013;186:36-44.

3. Boizard M, Le Liepvre X, Lemarchand P, Foufelle F, Ferré P, Dugail I. Obesity-related overexpression of fatty-acid synthase gene in adipose tissue involves sterol regulatory element-binding protein transcription factors. J Biol Chem. 1998:273:29164-71.

4. Clément K, Vaisse C, Manning BSJ, Basdevant A, Guy-Grand B, Ruiz J, et al. Genetic variation in the $\beta 3$-adrenergic receptor and an increased capacity to gain weight in patients with morbid obesity. N Engl J Med. 1995:333:352-4.

5. Coon SL, Mazuruk K, Bernard M, Roseboom PH, Klein DC, Rodriguez IR. The human serotonin $\mathrm{N}$-acetyltransferase (EC 2.3.1.87) gene (AANAT): structure, chromosomal localization, and tissue expression. Genomics. 1996;34:76-84.

6. Coon SL, Zarazaga LA, Malpaux B, Ravault JP, Bodin L, Voisin P, et al. Genetic variability in plasma melatonin in sheep is due to pineal weight, not to variations in enzyme activities. Am J Physiol Endocrinol Metab. 1999;227:792-7.

7. Da Costa A, Pires V, Fontes C, Mestre Prates J. Expression of genes controlling fat deposition in two genetically diverse beef cattle breeds fed high or low silage diets. BMC Vet Res. 2013;9:118.

8. Deckelbaum RJ, Worgall TS, Seo T. N -3 fatty acids and gene expression. The Am J Clin Nutr. 2006:83:S1520-5.

9. Higuchi R, Fockler C, Dollinger G, Watson R. Kinetic PCR analysis: Real-time monitoring of DNA amplification reactions. Biotech. 1993;11:1026-30.

10. Forrest RH, Hickford JGH, Frampton CM. Polymorphism at the ovine $\beta 3$-adrenergic receptor locus (ADRB3) and its association with lamb mortality. J Anim Sci. 2007:85:2801-6.

11. Forrest RH, Hickford JGH, Hogan A, Frampton C. Polymorphism at the ovine ß3-adrenergic receptor locus: associations with birth weight, growth rate, carcass composition and cold survival. Anim Genet. 2003;34:19-25.

12. Guo B, Kongsuwan K, Greenwood P, Zhou G, Zhang W, Dalrymple B. A gene expression estimator of intramuscular fat percentage for use in both cattle and sheep. J Anim Sci Biotech. 2014,5:35.

13. Holman B, Kashani A, Malau-Aduli A. Growth and body conformation responses of genetically divergent Australian sheep to Spirulina (Arthrospira platensis) supplementation. Am J Exp Agri. 2012;2:160-73.

14. Holman BWB, Kashani A, Malau-Aduli AEO. Effects of Spirulina (Arthrospira platensis) supplementation level and basal diet on liveweight, body conformation and growth traits in genetically divergent Australian dual-purpose lambs during simulated drought and typical pasture grazing. Small Rum Res. 2014;120:6-1.

15. Holman BWB, Malau-Aduli AEO. Spirulina as a livestock supplement and animal feed. J Anim Phys Anim Nut. 2013;97:615-23. 
16. Hu J, Zhou H, Smyth A, Luo Y, Hickford JG. Polymorphism of the bovine ADRB3 gene. Mol Bio Reports. 2010;37:3389-92.

17. Jiménez-Aranda A, Fernández-Vázquez G, Campos D, Tassi M, Velasco-Perez L, Tan DX, et al. Melatonin induces browning of inguinal white adipose tissue in Zucker diabetic fatty rats. J Pineal Res. 2013;55:416-23.

18. Kamaid A, Giráldez F. BTG1 and BTG2 gene expression during early chick development. Dev Dynamics. 2008;237:2158-69.

19. Mo XY, Lan J, Jiao QZ, Xiong YZ, Zuo B, Li FE, et al. Molecular characterization, expression pattern and association analysis of the porcine BTG2 gene. Mol Bio Reports. 2011;38:4389-96.

20. Perez R, Cañón J, Dunner S. Genes associated with long-chain omega-3 fatty acids in bovine skeletal muscle. J Appl Genet. 2010;51:479-87.

21. Pfaffl MW. A new mathematical model for relative quantification in real-time RT-PCR. Nucleic Acids Res. 2001;29:e45.

22. Reiter RJ, Tan DX, Galano A. Melatonin reduces lipid peroxidation and membrane viscosity: a review. J Pineal Res. 2014;56:225-37.

23. Roy R, Taourit S, Zaragoza P, Eggen A, Rodellar C. Genomic structure and alternative transcript of bovine fatty acid synthase gene FASN: comparative analysis of the FASN gene between monogastric and ruminant species. Cytogenet Genome Res. 2005;111:65-73.

24. Strosberg AD. Structure and function of the $\beta 3$-adrenergic receptor. Annu Rev Pharmacol and Toxicol. 1997;37:421-50

25. Wu J, Liu W, Liu J, Qiao L, Yuan Y. Distribution and quantification of $\beta-3$ adrenergic receptor in tissues of sheep. Animal. 2011:5:88-93.

26. Wu J, Qiao L, Liu J, Yuan Y, Liu W. SNP variation in ADRB3 gene reflects the breed difference of sheep populations. Mol Bio Reports. 2012;39:8395-403.

\section{Submit your next manuscript to BioMed Central and take full advantage of:}

- Convenient online submission

- Thorough peer review

- No space constraints or color figure charges

- Immediate publication on acceptance

- Inclusion in PubMed, CAS, Scopus and Google Scholar

- Research which is freely available for redistribution 